\title{
Counting edge-transitive, one-ended, three-connected planar maps with a given growth rate
}

\author{
Tomaž Pisanski \\ Faculty of Mathematics and Physics, University of Ljubljana \\ Jadranska 19, 1000 Ljubljana, Slovenia
}

Received 17 January 2008, accepted 4 September 2009, published online 30 September 2009

\begin{abstract}
B. Grünbaum and G. C. Shephard have classified one-ended, 3-connected, planar, edgetransitive maps. It turns out that each of these maps can be described uniquely by an edgesymbol $\langle p, q ; k, \ell\rangle$. Recently the growth rate of each of these maps has been determined by S. Graves, T. Pisanski and M. E. Watkins. We determine the number of edge-transitive maps for a given growth rate.
\end{abstract}

Keywords: Tessellation, edge-transitive, Bilinski diagram, exponential growth, enumeration.

Math. Subj. Class.: 05B45, 05C12, 52C20

Dedicated to Professor Ivan Vidav on the occasion of his 90th birthday.

\section{Introduction}

All maps in this article are planar, 3-connected, dually 3-connected and one-ended, i.e., the deletion of no finite subgraph leaves two or more infinite components. Our maps may be finite or infinite, but are locally finite, i.e., all valences and co-valences are finite. It is well known that for every such map, there exists a unique planar embedding.

We are interested in the "rate of growth" of such maps. Our measure of this rate of growth is the limit of the ratio $\Gamma(n):=\tau(n+1) / \tau(n)$ as $n$ approaches infinity, when such a limit exists, where $\tau(n)$ is the number of vertices in the first $n$ layers (also called coronas) of the Bilinski diagram. We follow [5] and call these maps (edge-transitive planar) tessellations.

In this article we are interested in obtaining explicit values for the number of edgetransitive tessellations with a given growth rate. In this case the underlying graph is edgehomogeneous, i.e., there exists a 4-tuple $\langle p, q ; k, \ell\rangle$ of integers $\geq 3$, called the edge-symbol

E-mail address: Tomaz.Pisanski@ fmf.uni-lj.si (Tomaž Pisanski) 
of the tessellation, such that for each edge, $p$ and $q$ are the valences of its two incident vertices and $k$ and $\ell$ are the covalences of its two incident faces.

It is shown in [9] that in case of edge-transitive planar tessellations the growth rate is independent of the root of the Bilinski diagram. Hence we may define the limit $\Gamma(M)=$ $\lim _{n \rightarrow \infty} \Gamma(n)$ without specifying which Bilinski diagram is used for computing $\Gamma(n)$. Recall that a tessellation $M$ is normal if each face is homeomorphic to a closed disk, any two faces are either disjoint or meet at a vertex or at an edge and there exist two radii $r$ and $R, r<R$, such that each face contains a ball of radius $r$ and is entirely contained in a ball of radius $R$. Let us define

$$
\mu(M):=\frac{1}{p}+\frac{1}{q}+\frac{1}{k}+\frac{1}{\ell} .
$$

Assume that the tessellation $M$ with the edge symbol $\langle p, q ; k, \ell\rangle$ exists. It is well-known that if $\mu(M)>1$, then the tessellation $M$ is finite and has a normal realization on the sphere. More interesting is that when $\mu(M)=1$, then the tessellation has a normal realization in the Euclidean plane $E^{2}, \tau(n)$ grows quadratically, and $\Gamma(M)=1$. However, when $\mu(M)<1$, then the tessellation has a normal realization in the hyperbolic plane $H^{2}, \tau(n)$ grows exponentially, and $\Gamma(M)>1$.

One would expect that the tessellations with higher growth rate would have smaller value of parameter $\mu$. This is true for most cases. However, there are numerous counterexamples. For instance, $\Gamma(\langle 3,8 ; 4,4\rangle)>\Gamma(\langle 4,5 ; 4,4\rangle)$ while $\mu(\langle 3,8 ; 4,4\rangle)>\mu(\langle 4,5$; $4,4\rangle)$; see [5], Table 1 . It seems that there is no mapping $F$ that would allow us to compute $\Gamma$ if $\mu$ is known: $\Gamma(\langle p, q ; k, \ell\rangle)=F(\mu(\langle p, q ; k, \ell\rangle))$. We could not find any mapping $F^{\prime}$ that would express $\mu$ in terms of $\Gamma: \mu(\langle p, q ; k, \ell\rangle)=F^{\prime}(\Gamma(\langle p, q ; k, \ell\rangle))$. This means that the connection between the growth rate $\Gamma(M)$ and parameter $\mu(M)$ is much more involved and is only partially explained in [5].

\section{Preliminaries}

For the tessellations considered in this article, Grünbaum and Shephard [7] have obtained the following strong result rewritten according to our needs.

Proposition 2.1. [7] There exists an edge-transitive tessellation with valences $p$ and $q$ and covalences $k$ and $\ell$ if and only if $p, q, k, \ell$ are integers such that $3 \leq p \leq q$ and $3 \leq k \leq \ell$ and exactly one of the following holds:

(1) all of $p, q, k, \ell$ are even;

(2.1) $k=\ell$ even, and exactly one of $p, q$ is odd;

(2.2) $k=\ell$ even, and both $p, q$ are odd;

(3.1) $p=q$ even, and exactly one of $k, \ell$ is odd;

(3.2) $p=q$ even, and both $k, \ell$ are odd;

(4) $p=q, k=\ell$, and all are odd.

The parameters $p, q, k, \ell$ determine the tessellation up to homeomorphism of the plane.

Note that in the original formulation of the theorem, the cases (2.1) and (2.2) are combined into

(2) $k=\ell$ even, and at least one of $p, q$ is odd;

and similarly the cases (3.1) and (3.2) into

(3) $p=q$ even, and at least one of $k, \ell$ is odd.

If the parameters $p, q, k$, and $\ell$ satisfy the conditions of Proposition 2.1 we call the corresponding edge symbol $\langle p, q ; k, \ell\rangle$ admissible. The result of Grünbaum and Shephard 
shows in the one-ended case not only that each edge-homogeneous tessellation is also edgetransitive but also that edge-transitive tessellations are in one-to-one correspondence with admissible edge-symbols. We remark that for a given edge-symbol, there may exist more than one multi-ended map, but there also may exist none at all, as shown in [4].

In [5] the growth rate of edge-transitive tessellations is computed.

Definition 2.2. Let $M$ be a tessellation that is rooted at some vertex $v$. Define a sequence of sets $\left\{U_{n}: n \geq 0\right\}$ of vertices and a sequence of sets $\left\{F_{n},: n \geq 1\right\}$ of faces as follows:

- Let $U_{0}=\{v\}$ and let $F_{0}=\emptyset$;

- For $n \geq 1$, let $F_{n}$ denote the set of faces of $M$ not in $F_{n-1}$ that are incident with some vertex in $U_{n-1}$;

- For $n \geq 1$, let $U_{n}$ denote the set of vertices of $M$ not in $U_{n-1}$ that are incident with some face in $F_{n}$.

The stratification $\left\{U_{n}\right\}$ and $\left\{F_{n}\right\}$ is called the Bilinski diagram $B_{v}$ of $M$ rooted at $v$.

In a similar way one could define the Bilinski diagram $B_{f}$ of $M$ rooted at $f$; see [5].

These diagrams were first used by Stanko Bilinski in his dissertation [1, 2] and later also by others such as Grünbaum and Shephard [8]. In [10] they were formally introduced as "Bilinski maps".

Intuitively, most tessellations can be labeled as Bilinski diagrams by arbitrarily selecting a vertex to comprise the singleton set $U_{0}$ and then calling by $U_{n}$ each set of vertices on subsequent concentric circles with (increasing) radius $n$. The annulus between two consecutive concentric circles $\left\langle U_{n-1}\right\rangle$ and $\left\langle U_{n}\right\rangle$ is partitioned by the set of faces $F_{n}$. Thus the neighborhood of a vertex in $U_{n}$ is contained in $U_{n-1} \cup U_{n} \cup U_{n+1}$.

A Bilinski diagram is concentric if each subgraph induced by $U_{n}(n \geq 1)$ is a circuit. If a tessellation yields a concentric Bilinski diagram regardless of which vertex or face is chosen as its root, then the tessellation is uniformly concentric.

For edge-transitive tessellations it is possible to verify that a tessellation either is uniformly concentric or is not concentric for any given root. Furthermore, the tessellation is concentric if and only if its dual is concentric.

Now we can give a precise definition of the function $\tau(n)$ defined in the previous section. Let the tessellation $M$ be labeled as a Bilinski diagram. Then

$$
\tau(n):=\sum_{j=0}^{n}\left|U_{n}\right|, \quad n=0,1, \ldots,
$$

and the growth rate of $M$ is defined as

$$
\Gamma(M)=\lim _{n \rightarrow \infty} \frac{\tau(n+1)}{\tau(n)}
$$

if this limit exists.

In [5] S. Graves, T. Pisanski and M. E. Watkins determined the growth rate $\Gamma(M)$ of any tessellation with the admissible edge-symbol $\langle p, q ; k, \ell\rangle$. It turns out that the growth rate can be expressed in terms of a single parameter

$$
t:=((p+q) / 2-2)((k+\ell) / 2-2) .
$$

Let us define the function

$$
G(t)=(t-2+\sqrt{t(t-4)}) / 2 .
$$


Proposition 2.3. ([3]) Let $M$ be an edge-transitive tessellation with the admissible edgesymbol $\langle p, q ; k, \ell\rangle, 3 \leq p \leq q$ and $3 \leq k \leq \ell$. Then

(a) if $p=3, q \geq 6, k=\ell=4$, then neither $M$ nor its dual are concentric;

(b) in all other cases both $M$ and its dual are uniformly concentric.

Proposition 2.4. ([5]) Let $M$ be an edge-transitive tessellation with the admissible edgesymbol $\langle p, q ; k, \ell\rangle$ or $\langle k, \ell ; p, q\rangle, 3 \leq p \leq q$ and $3 \leq k \leq \ell$. Then

$$
\Gamma(M)=\Gamma(t)= \begin{cases}\mathrm{G}(\mathrm{t}-1) & \text { if the tessellation } M \text { is not concentric, } \\ \mathrm{G}(\mathrm{t}) & \text { if the tessellation } M \text { is concentric. }\end{cases}
$$

There are nine finite tessellations not covered by this Proposition. There are also five Euclidean tessellations with growth rate 1 . All other growth rates are exponential and are given by the set $\{G(t) \mid t=5,6, \ldots\}$ and are irrational numbers.

\section{Counting tessellations with a given growth rate}

In this section we will count the number of edge-transitive tessellations with a given growth rate. Let $a_{0}:=9$ denote the number of finite tessellations, $a_{1}$ the number of Euclidean tessellations (growth rate $\Gamma_{1}:=1$ ), $a_{2}$ the number of tessellations with the smallest exponential growth rate

$$
\Gamma_{2}:=G(5)=(3+\sqrt{5}) / 2
$$

and in general, for any $n \geq 2$ let $a_{n}$ count the number of tessellations with the growth rate $\Gamma_{n}=G(n+3)$.

Before we state the main result let us recall some divisor functions. Let $\sigma_{k}(n)$ denote the sum of $k$-th powers of divisors of a positive integer $n: \sigma_{k}(n)=\sum_{d \mid n} d^{k}$. Similarly, let $\sigma_{k}^{o}(n)$ denote the sum of $k$-th powers of odd divisors of the integer $n$.

Let $N(t)$ denote the number of tessellations with parameter $t=((p+q) / 2-2)((k+$ $\ell) / 2-2)$ for which the edge-symbol $\langle p, q ; k, \ell\rangle$ is admissible. First we establish the number of tessellations $N(t)$ for $t \geq 4$.

Theorem 3.1. The number of tessellations with parameter $t$ is given by:

(a) $N(t)=\frac{t}{4} \sigma_{0}\left(\frac{t}{4}\right)+\frac{t}{2}\left(\sigma_{0}^{o}(t)-\sigma_{-1}^{o}(t)\right)+2 \sigma_{1}^{o}(t)+2 \sigma_{1}\left(\frac{t}{4}\right)$, if $t$ is divisible by 4 .

(b) $N(t)=\frac{t}{2}\left(\sigma_{0}\left(\frac{t}{2}\right)-\sigma_{-1}\left(\frac{t}{2}\right)\right)+2 \sigma_{1}\left(\frac{t}{2}\right)$, if $t$ is even but $\frac{t}{2}$ is odd.

(c) $N(t)=\frac{t+1}{4} \sigma_{0}(t)+\frac{1}{2} \sigma_{1}(t)$, if $t$ is odd.

Proof. In order to prove the result we have to count the number of non-isomorphic admissible edge-symbols $\langle p, q ; k, \ell\rangle$ with a given parameter $t$. Let us write $a=(p+q) / 2-2$ and $b=(k+\ell) / 2-2$. Clearly $a$ and $b$ may be half-integers. However, if one is a half-integer, then the other one must be an even integer, making their product $t=a b$ an integer. We count separately each of the cases of Proposition 2.1: $N(t)=N_{1}(t)+$ $N_{2}(t)+N_{3}(t)+N_{4}(t)$ and $N_{2}(t)=N_{21}(t)+N_{22}(t), N_{3}(t)=N_{31}(t)+N_{32}(t)$. Note that the subscripts of $N$ introduced here are in one-to-one correspondence with each case or subcase of Proposition 2.1. For instance, $N_{22}(t)$ counts the number of tessellations with $t=((p+q) / 2-2)((k+\ell) / 2-2)$ such that $3 \leq p \leq q, 3 \leq k \leq \ell$ where $k=\ell$ is even, and both $p, q$ are odd.

Case 1. If $p=2 p_{0}, q=2 q_{0}, k=2 k_{0}, \ell=2 \ell_{0}$ are all even numbers, then $p_{0}+q_{0}=$ $a+2, k_{0}+\ell_{0}=b+2$, where $p_{0}, q_{0}, k_{0}, \ell_{0} \geq 2$. For any choice of $p_{0}$ and $k_{0}$ such that 
$2 \leq p_{0} \leq(a+2) / 2$ and $2 \leq k_{0} \leq(b+2) / 2$ we obtain a different tessellation. For a given divisor $a$ of $t$ there are $\lfloor a / 2\rfloor\lfloor b / 2\rfloor$ choices. Therefore the total number of such tessellations is given by:

$$
N_{1}(t):=\sum_{a \mid t}\lfloor a / 2\rfloor\lfloor t /(2 a)\rfloor
$$

Case 2. Here $k=\ell$ is an even integer. In this case $b=k-2$ is even. There are two subcases.

Case 2.1. First we determine the number of admissible edge-symbols with $p+q$ odd. In this case $r=2 a=p+q-4$ is an odd divisor of $t$ and the number of such tessellations is given by the formula:

$$
N_{21}(t):=\sum_{r \mid t, r \text { odd }}(r-1) / 2 .
$$

Case 2.2. In this case both $p$ and $q$ are odd. This can happen only if $t$ is even. In the following formula for $N_{22}$, the first summation covers the case $p \neq q$ while the second one counts the admissible edge symbols with $p=q$.

$$
N_{22}(t):= \begin{cases}0 & \text { if } t \text { is odd, } \\ \sum_{r \mid(t / 2)}\lfloor t /(4 r)\rfloor+\sum_{r \mid t, r} \text { odd } 1 & \text { if } t \text { is even. }\end{cases}
$$

Case 3. Due to the symmetry, the counts are the same as in Case 2:

$$
N_{31}(t)=N_{21}(t), N_{32}(t)=N_{22}(t)
$$

Case 4. Finally, we determine the number of admissible edge-symbols with $k=\ell$ and $p=q$, all odd. This can happen only in case $t$ is odd.

$$
N_{4}(t):= \begin{cases}0 & \text { if } t \text { is even } \\ \sum_{r \mid t} 1 & \text { if } t \text { is odd. }\end{cases}
$$

Hence:

$$
N(t)=N_{1}(t)+2 N_{21}(t)+2 N_{22}(t)+N_{4}(t)
$$

where $N_{1}(t), N_{21}(t), N_{22}(t)$, and $N_{4}(t)$ are expressed as summations above. After some algebraic computations the result follows. In these computations we use the results of Lemma 3.2.

Lemma 3.2. The numbers $N_{1}(t), N_{2}(t), N_{3}(t), N_{4}(t)$ of planar edge-transitive tessellations with parameter $t$, satisfying one of the conditions $1-4$ of the Proposition 2.1 are given by:

(1a) $N_{1}(t)=\frac{t}{4} \sigma_{0}(t / 4)+\frac{t}{2}\left(\sigma_{0}^{o}(t)-\sigma_{-1}^{o}(t)\right)$, if $t$ is divisible by 4 .

(1b) $N_{1}(t)=\frac{t}{2}\left(\sigma_{0}^{o}(t)-\sigma_{-1}^{o}(t)\right)$, if $t$ is even but $\frac{t}{2}$ is odd.

(1c) $N_{1}(t)=\frac{t+1}{4} \sigma_{0}(t)-\frac{1}{2} \sigma_{1}(t)$, if $t$ is odd.

(2.1) $N_{21}(t)=\frac{1}{2}\left(\sigma_{1}^{o}(t)-\sigma_{0}^{o}(t)\right)$.

(2.2a) $N_{22}(t)=\sigma_{1}\left(\frac{t}{4}\right)+\frac{1}{2}\left(\sigma_{1}^{o}(t)+\sigma_{0}^{o}(t)\right)$, if $t$ is divisible by 4 .

(2.2b) $N_{22}(t)=\frac{1}{2}\left(\sigma_{1}^{o}(t)+\sigma_{0}^{o}(t)\right)$, if $t$ is even but $\frac{t}{2}$ is odd.

(2.2c) $N_{22}(t)=0$, if $t$ is odd. 
(3.1) $N_{31}(t)=\frac{1}{2}\left(\sigma_{1}^{o}(t)-\sigma_{0}^{o}(t)\right)$.

(3.2a) $N_{32}(t)=\sigma_{1}\left(\frac{t}{4}\right)+\frac{1}{2}\left(\sigma_{1}^{o}(t)+\sigma_{0}^{o}(t)\right)$, if $t$ is divisible by 4 .

(3.2b) $N_{32}(t)=\frac{1}{2}\left(\sigma_{1}^{o}(t)+\sigma_{0}^{o}(t)\right)$, if $t$ is even but $\frac{t}{2}$ is odd.

(3.2c) $N_{32}(t)=0$, if $t$ is odd.

(4a) $N_{4}(t)=0$, if $t$ is divisible by 4 .

(4b) $N_{4}(t)=0$, if $t$ is even but $\frac{t}{2}$ is odd.

(4c) $N_{4}(t)=\sigma_{0}(t)$, if $t$ is odd;

where $N_{2}(t)=N_{21}(t)+N_{22}(t), N_{3}(t)=N_{31}(t)+N_{32}(t)$, and $N(t)=N_{1}(t)+$ $N_{2}(t)+N_{3}(t)+N_{4}(t)=N_{1}(t)+2 N_{2}(t)+N_{4}(t)$.

Proof. The cases (2.2c), (4a) and (4b) are obtained by inspection. Case (4c) follows from the fact that for $t$ odd by definition $N_{4}(t)=\sum_{a \mid t} 1=\sigma_{0}(t)$. Cases (3.1), (3.2a),(3.2b) and (3.2c) follow by symmetry from the corresponding cases (2.1), (2.2a),(2.2b) and (2.2c). Hence we are only left with non-trivial cases (1a),(1b),(1c),(2.1),(2.2a),(2.2b) and (2.2c). We show how to do case (1c), the rest follows along the same line of reasoning. Recall that

$$
N_{1}(t)=\sum_{a \mid t}\lfloor a / 2\rfloor\lfloor t /(2 a)\rfloor
$$

We may split the summation into 4 parts: the one (i) in which both $a$ and $b=t / a$ are even,(ii) $a$ even, $b$ odd, (iii) $a$ odd, $b$ even and (iv) both $a$ and $b$ odd. The first case (i) is non-zero only if $t$ is divisible by four, the second (ii) and third (iii) are equal and are non-zero only if $t$ is even while the last case (iv) is non-zero only if $t$ is odd. For $t$ odd we readily get

$$
N_{1}(t)=\sum_{a \mid t}((a-1) / 2)((t / a-1) / 2)=\sum_{a \mid t}(t-t / a-a+1) / 4 .
$$

It is not hard to deduce the result (1c) $N_{1}(t)=\frac{t+1}{4} \sigma_{0}(t)-\frac{1}{2} \sigma_{1}(t)$ for $t$ odd. Note that (i) contributes only to (1a) while (ii) contributes both to (1a) and (1b).

Using this result it is now possible to produce a table.

$$
\begin{array}{llllllllllllll}
t & 1 & 2 & 3 & 4 & 5 & 6 & 7 & 8 & 9 & 10 & 11 & 12 & \ldots \\
N(t) & 1 & 2 & 4 & 5 & 6 & 10 & 8 & 12 & 14 & 16 & 12 & 26 & \ldots
\end{array}
$$

This table is useful in computing the number of edge-transitive tessellations with a given growth rate.

Corollary 3.3. The number of planar edge-transitive tessellations $a_{n}$ with the $n$-th growth rate is determined by: $a_{n}=N(n+3), n \geq 1$.

Proof. Note that $n=1$ corresponds to the Euclidean case, $n=2$ to the hyperbolic case with the minimal growth, etc. This seemingly trivial consequence of Theorem 3.1 has a subtle twist, since not all tessellations for a given parameter $t \geq 4$ have the same growth rate $G(t)$. Exactly two of them, namely $\langle 3, t-1 ; 4,4\rangle$ and its dual $\langle 4,4 ; 3, t-1\rangle$, have growth rate $G(t-1)$. This is due to the fact that these two tessellations have non-concentric Bilinski diagrams. But the total number remains $N(t)$, since we lose two tessellations at level $t$, but gain two similar tessellations at level $t+1$. The argument therefore works for all infinite tessellations. The finite ones are counted separately. 
Using the formula that follows from Theorem 3.1 and Corollary 3.3 one can now construct the table that counts the number of non-isomorphic tessellations with a given growth rate. Here we list the smallest values of the sequence $a_{n}$ together with the corresponding growth rates.

$\begin{array}{lllllllllll}n & 0 & 1 & 2 & 3 & 4 & 5 & 6 & 7 & 8 & \ldots \\ a_{n} & 9 & 5 & 6 & 10 & 8 & 12 & 14 & 16 & 12 & \ldots \\ \Gamma_{n} & - & 1 & 2.61803 & 3.73205 & 4.79129 & 5.82843 & 6.85410 & 7.87298 & 8.88748 & \ldots\end{array}$

It is natural to look for a positive constant $c$ and a suitable function $f(t)$ such that for any integer $t>2$ the function $N(t)$ may be bounded: $t \leq N(t) \leq c t f(t)$. Our result expresses $N(t)$ as a sum of several divisor functions. In order to determine the correct asymptotic value we need to find the one with the largest growth. Gronwall's theorem states that $\limsup _{n \rightarrow \infty} \sigma_{1}(n) / n \ln \ln n=e^{\gamma}$, where $\gamma$ is Euler's constant; see [6]. This suggested that $f(t)=\ln \ln t$. For $3 \leq t \leq 100000$ one can take $c=4 /(3 \ln (\ln 3))<15$. However, for large values of $t$ this inequality does not hold, actually, it fails badly, as pointed out by one referee. The upper bound with $c=15$ is violated, for instance, for $t=2^{300}$. A more reasonable bound seems to hold for $f(t)=\ln t$. This bound may be derived, for instance, for $t=p^{k}$, where $p$ is a prime. Whether this bound is indeed the correct upper bound for any integer $t \rightarrow \infty$ remains to be proved.

\section{Acknowledgements}

The author would like to thank Steve Graves and Mark Watkins for valuable remarks, to the referees for considerably improving the presentation, and to Karin Cvetko Vah for proofreading the formulae.

\section{References}

[1] S. Bilinski, Homogene mreže ravnine, Rad Jugoslav. Akad. Znanosti i Umjetnosti 271 (1948), 145-255.

[2] S. Bilinski, Homogene Netze der Ebene, Bull. Internat. Acad. Yougoslave. Cl. Sci. Math. Phys. Tech. (N.S.) 2 (1949), 63-111.

[3] J. A. Bruce and M. E. Watkins, Concentric Bilinski diagrams, Australasian J. Combin. 30 (2004), 161-174.

[4] J. E. Graver and M. E. Watkins, Locally Finite, Planar, Edge-Transitive Graphs, Memoirs of the Amer. Math. Soc. 126 (1997), no. 601.

[5] S. Graves, T. Pisanski and M. E. Watkins, Growth of Edge-Homogeneous Tessellations, SIAM J. Discrete Math. 23 (2008), 1-18.

[6] T. H. Gronwall, Some Asymptotic Expressions in the Theory of Numbers, Trans. Amer. Math. Soc. 14 (1913), 113-122.

[7] B. Grünbaum and G. C. Shephard, Edge-transitive planar graphs, J. Graph Theory 11 (1987), 141-155.

[8] B. Grünbaum and G. C. Shephard, Tilings and Patterns, W. H. Freeman and Company, New York, 1987.

[9] J. F. Moran, The growth rate and balance of homogeneous tilings in the hyperbolic plane, Discrete Math 173 (1997), 151-186. 
[10] P. Niemeyer and M. E. Watkins, Geodetic rays and fibers in one-ended planar graphs. J. Combin. Theory B 69 (1997), 142-163. 